\title{
Diffuse left ventricular interstitial fibrosis is associated with sub-clinical myocardial dysfunction in Alström Syndrome: an observational study
}

Nicola C. Edwards ${ }^{1,2^{*}}$, William E. Moody ${ }^{1,2}$, Mengshi Yuan ${ }^{1}$, Adrian T. Warfield ${ }^{3}$, Robert Cramb ${ }^{4}$, Richard B. Paisey ${ }^{5}$, Tarekegn Geberhiwot ${ }^{6}$ and Richard P. Steeds ${ }^{1,2}$

\begin{abstract}
Background: Alström syndrome is a rare inherited ciliopathy with progressive multisystem involvement. Dilated cardiomyopathy is common in infancy and recurs or presents de novo in adults with high rates of premature cardiovascular death. Although Alström syndrome is characterised by fibrosis in solid organs such as the liver, the pathogenesis of related cardiomyopathy are not clear. To date it is not known whether diffuse interstitial myocardial fibrosis is present before the onset of heart failure symptoms or changes in conventional parameters of left ventricular function.

Methods: In this observational study, 26 patients with Alström syndrome (mean age $27 \pm 9$ years, $65 \%$ male, $24 \mathrm{~h}$ ABPM $130 \pm 14$ / $77 \pm 9 \mathrm{mmHg}$ ) without symptomatic cardiovascular disease were recruited from a single centre and compared to matched healthy controls. All subjects underwent cardiac MRI (1.5 T) to assess ventricular function, diffuse interstitial myocardial fibrosis by measurement of extracellular volume on T1-mapping (MOLLI) and coarse replacement fibrosis using standard late gadolinium enhancement imaging.

Results: Global extracellular volume was increased in Alström syndrome with wider variation compared to controls ( $0.30 \pm 0.05$ vs. $0.25 \pm 0.01, p<0.05)$. Left ventricular long axis function and global longitudinal strain were impaired in Alström syndrome without change in ejection fraction, ventricular size or atrial stress (NT-proBNP) $(p<0.05)$. Global extracellular volume was associated with reduced peak systolic longitudinal strain $(r=-0.73, p<0.01)$ and strain rate $(r=-0.57, p<0.01)$, increased QTc interval $(r=0.49, p<0.05)$ and serum triglycerides $(r=0.66, p<0.01)$. Nine $(35 \%)$ patients had diffuse mid-wall late gadolinium enhancement in a non-coronary artery distribution.

Conclusion: Diffuse interstitial myocardial fibrosis is common in Alström syndrome and is associated with impaired left ventricular systolic function. Serial studies are required to determine whether global extracellular volume may be an independent imaging biomarker of vulnerability to dilated cardiomyopathy and heart failure.
\end{abstract}

Keywords: Alström syndrome, Myocardial fibrosis, Cardiac MRI, T1 mapping, Left ventricular function

\section{Background}

Alström syndrome (ALMS) is a rare autosomal recessive syndrome (OMIM 203800) characterised by childhood onset retinal cone rod dystrophy, neuronal hearing loss, obesity and severe insulin resistance presenting with exaggerated features of the metabolic syndrome [1-4].

\footnotetext{
* Correspondence: n.c.edwards@bham.ac.uk

${ }^{1}$ School of Clinical and Experimental Medicine, University of Birmingham, Birmingham, UK

2Department of Cardiology, Queen Elizabeth Hospital, Birmingham, UK Full list of author information is available at the end of the article
}

Estimates of the prevalence range from 1:10,000 [5] to fewer than 1:1,000,000 [6]. About 800 individuals diagnosed with ALMS have been identified worldwide. Idiopathic infantile dilated cardiomyopathy (ICM) presents acutely in nearly half of all ALMS subjects within the first few weeks of life $[7,8]$. In survivors, the pathological process then appears to be fully reversible, as there are no persisting clinical, electrocardiographic or echocardiographic evidence of ventricular dysfunction, nor is there evidence of coarse myocardial fibrosis [8]. Cardiomyopathy may then recur or develop de novo in up to $65 \%$ of adolescents and adults

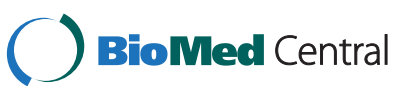


with high rates of morbidity and mortality, at which stage coarse fibrosis is evident on post-mortem [9]. This has also been demonstrated as patchy late gadolinium enhancement (LGE) using cardiac magnetic resonance imaging (CMR) $[10,11]$. Although ALMS is characterised by diffuse fibrosis in other solid organs, there are only limited data identifying whether diffuse interstitial fibrosis may be present in the myocardium before onset of dilated cardiomyopathy [10]. Diffuse interstitial fibrosis may be missed on conventional late gadolinium enhancement (LGE) imaging, which relies on comparison of signal intensities between normal and abnormal myocardium within the same image [12]. This limitation is overcome with newer CMR T1-mapping techniques which can quantify the size of the extracellular volume (ECV). The primary aims of this study were; 1 ) to establish whether diffuse interstitial myocardial fibrosis is present in asymptomatic adult subjects with ALMS, 2) if present, determine whether this is associated with change in left ventricular function.

The profound metabolic disturbances which characterise ALMS include severe insulin resistance, activation of the renin angiotensin aldosterone system and raised serum triglycerides - each of which has a potential role in the development of myocardial fibrosis [2, 13-15]. Thus, although ALMS is rare, it offers a model of disease processes, including metabolic syndrome, that are of increasing importance to a much wider population. A secondary aim of this study was to identify associations between potential metabolic changes in ALMS that might mediate myocardial fibrosis.

\section{Methods \\ Study design}

A propsoective cohort observational study performed between March 2012 and November 2013.

\section{Patients \& setting}

In total, 26 consecutive adults with genetically proven ALMS attending the National Centre for Rare Disease at the Queen Elizabeth Hospital Birmingham, England were studied prospectively as part of standard clinical care. Two other patients were seen at the National Centre during this time period but did not proceed with CMR due to contra-indications (one permanent pacemaker; one with a cochlear implant). Patients were compared to gender and age matched healthy controls with no history of cardiac disease recruited as part of an on-going clinical study [16].

\section{Ethics statement}

This study was approved by the National Research Ethics Service and all patients provided written informed consent to participate (12/WM/0250).

\section{Cardiac MRI}

Consent was obtained for contrast enhanced CMR (1.5 T Avanto, Siemens Healthcare, Erlangen, Germany). Left ventricular (LV) dimensions, volumes and mass were acquired in line with standard CMR protocols [17]. Mitral annular plane systolic excursion (MAPSE) were measured on standard 4-chamber SSFP cine images, using the straight-line distance travelled by the lateral mitral annulus from end-diastole to end-systole. CMR-tagging (SPAMM) was performed in the horizontal long-axis (4 chamber) image using prospective electrocardiographic gating [18]. Late enhancement (LGE) imaging was performed 7-10 $\mathrm{min}$ after $0.15 \mathrm{mmol} / \mathrm{Kg}$ of gadolinium contrast bolus (Gadovist Bayer Health Care). LGE is an imaging technique that characterizes the myocardium by detecting patterns of disease including reparative fibrosis and infarction. Differences in the "wash out" kinetics of damaged myocardium creates relative differences in signal intensity within the myocardium to highlight areas that are abnormal (white) compared to areas that are normal (black). These white areas correspond to macroscopic reparative fibrosis. The technique is reliant on the heart containing areas of normal myocardium to reveal the difference in signal intensity - which is one reason why the technique may fail to detect diffuse myocardial disease throughout the ventricle. T1 mapping is a newer technique which has the advantage that there is no need for "normal" myocardium against which to compare $\mathrm{T} 1$ values within the same heart and is thus capable of detecting diffuse fibrosis, as has been shown in other conditions [9] and is postulated in ALMS. Therefore, in addition to standard LGE, short-axis images at LV basal and mid-ventricular levels were acquired using an ECG-gated modified Look-Locker inversion recovery sequence (MOLLI) sequence for T1 mapping before contrast and at 15-20 min after bolus contrast administration [19]. No patient had complications arising from the use of Gadolinium contrast.

\section{CMR image analysis}

Analysis was performed off-line using Argus Software $\left(\right.$ Siemens $^{\circ}$ ) according to the Society for Cardiovascular MR guidelines for reporting CMR with parameters indexed to height and body surface area where appropriate [20]. Tagged images were analyzed with CIMTag2D software (Cardiac Image Modelling, University of Auckland, Auckland) to calculate global peak systolic strain, peak systolic strain rate (myocardial deformation) and peak early diastolic strain rate (an indicator of diastolic function) [18].

Quantitative parametric images of myocardial ECV were generated with manual contouring to define a region of interest (ROI) within the LV myocardium in the ventricular septum (4-chamber view) and at basal and mid ventricular level (short-axis) on matched pre and post contrast images 
(Siemens, Erlangen, Germany). Care was taken to avoid contaminating T1 measurements at the myocardial-blood pool interface. T1 time in each ROI was recorded and used to calculate myocardial extracellular volume (ECV) by validated formulae [21].

$$
\mathrm{ECV}=((\Delta \mathrm{R} 1 \text { myocardium }) /(\Delta \mathrm{R} 1 \text { blood pool }))(1-\mathrm{Hct})
$$

HCT refers to the haematocrit recorded on a venous blood sample at the time of scan, $\Delta \mathrm{R} 1=1 / \mathrm{T} 1$ post contrast $-1 / \mathrm{T} 1$ pre contrast. The ECV was assessed in the left ventricle from the basal and mid ventricular levels, averaged to yield a "global ECV" measurement. Further details on the methods used have been published [21, 22].

\section{Biochemistry}

Venous blood samples were collected for routine haematology, biochemistry and lipid profiles. Serum N-terminal pro B Natriuretic peptide (NT-proBNP, ng/L) was measured by sandwich immunoassay with magnetic particle separation and chemiluminescent detection on an E170 analyser (Roche Diagnostics, Burgess Hill) with a lower limit of detection of $0.6 \mathrm{pmol} / \mathrm{L}$. Plasma renin was measured using an immunoradiometric assay (Cis Bio, IBA Molecular UK, Guildford) with age and position reference intervals and a detection limit of $5 \mathrm{ng} / \mathrm{L}$. Aldosterone was measured by competitive radioimmunoassay (Coat-a-Count, Siemens Medical Solutions Diagnositics, Llanberis.) which has reference intervals dependent on position with a detection limit of $60 \mathrm{pmol} / \mathrm{L}$. C-peptide $(\mathrm{pmol} / \mathrm{L}) /$ insulin $(\mathrm{pmol} / \mathrm{L})$ were also measured (Royal Surrey County Hospital, Guildford).

\section{Blood pressure}

Subjects underwent $24 \mathrm{~h}$ ambulatory blood pressure monitoring using a British Society of Hypertension approved protocol [23].

\section{Electrocardiogram}

Standard 12-lead electrocardiograms were recorded in each subject within $24 \mathrm{~h}$ of the CMR scan, with measurement of PR interval, QRS duration and QTc intervals using standard methodology (Bazett's formula for QTc).

\section{Statistical analysis}

Continuous variables are expressed as mean \pm standard deviation (SD) if normally distributed or median (interquartile range) if non-normally distributed by the Shapiro-Wilk test. Paired group comparisons were performed using independent T-test, Mann-Whitney and one-way analysis if variance with Bonferroni post hoc tests, as appropriate. Correlations between variables were assessed by Pearson if normally distributed and Spearman analyses if non-normally distributed. A multivariable regression model was used to assess predictors of ECV using variables associated with myocardial fibrosis. Statistical tests were two tailed and a p-value $<0.05$ was considered to indicate statistical significance.

\section{Results}

In total, 26 ALMS patients were compared with healthy controls (mean age $27 \pm 9$ vs. $27 \pm 6$ years, $p=0.24$ ). Subject genetic and demographic profiles are presented in Table 1 and Table 2 respectively. A history of infantile cardiomyopathy was documented in eight ALMS patients. One patient had end-stage renal disease (ESRD) requiring dialysis. One patient had a history of prior myocardial infarction. All patients were NYHA class I.

Patients with ALMS demonstrated marked metabolic derangements (Table 2). All were insulin resistant. Seventeen patients had diabetes and five patients had impaired glycaemia. Mean C-peptide: Glucose ratio (a marker of insulin resistance) was increased. Serum triglyceride levels were also elevated with reduced serum HDL levels compared to controls. NT-proBNP was increased above normal range ( $>200 \mathrm{ng} / \mathrm{L})$ in only 2 patients. One of these patients had ESRD and the subject was below the threshold for diagnosis of heart failure $(<400 \mathrm{ng} / \mathrm{L})$.

\section{Cardiovascular structure, function \& tissue characterization Ventricular volumes and function}

Patients with ALMS had reduced absolute and indexed LV volumes compared to controls but did not differ when indexed for height (to allow for obesity). Absolute LV mass did not differ between groups but was increased when indexed to height ${ }^{2.7}$. There was no difference in LV ejection fraction (EF) but longitudinal function (MAPSE) was reduced (Table 3).

\section{Myocardial tagging}

Left ventricular global longitudinal peak systolic strain and strain rate were reduced in ALMS (Table 3).

\section{T1 mapping}

Mean global ECV was increased compared to controls (Table 3) but with wider variation and cross-over with the normal population at the lower end of the range (Fig. 1). Patients with ALMS had reduced LV longitudinal function (MAPSE $12 \mathrm{~mm} \pm 2$ vs. $17 \mathrm{~mm} \pm 2, \mathrm{p}<0.01$ ), reduced global longitudinal strain $(11.2 \% \pm 2.6$ vs. $14.7 \% \pm 2.3$, $\mathrm{p}<0.01)$, strain rate $\left(0.58 \mathrm{~s}^{-1} \pm 0.11\right.$ vs. $0.83 \mathrm{~s}^{-1} \pm 0.15$, $\mathrm{p}<0.01)$ and reduced early diastolic strain rate $(0.49 \pm$ $0.18 \mathrm{~s}^{-1}$ vs. $\left.0.62 \pm 0.15 \mathrm{~s}^{-1}, \mathrm{p}<0.05\right)$. Fourteen patients (54 \%) had an ECV above the upper limit (0.279) for controls. Five patients had ECV above the upper limit 
Table 1 Individual patient demographic profile

\begin{tabular}{|c|c|c|c|c|c|c|c|c|c|}
\hline Patient & Age & Gender & Ethnicity & BMl & $\begin{array}{l}\text { ALMS } \\
\text { mutation }\end{array}$ & $\begin{array}{l}\text { Protein } \\
\text { change }\end{array}$ & $\begin{array}{l}\text { ALMS } \\
\text { mutation }\end{array}$ & $\begin{array}{l}\text { Protein } \\
\text { change }\end{array}$ & $\begin{array}{l}\text { Infantile } \\
\text { CM }\end{array}$ \\
\hline 1 & 21 & Male & White British & 32.6 & c. $6526 C>T$ & p.Gln2176X & C. $11101 C>T$ & p.Arg3701X & Yes \\
\hline 2 & 19 & Male & White British & 27.1 & c. $6526 \mathrm{C}>\mathrm{T}$ & p.Gln2176X & C. $11101 C>T$ & p.Arg3701X & No \\
\hline 3 & 18 & Male & White British & 32.3 & c. $8932 C>T$ & p.GLN2978X & $c .5356 A>G$ & p.ASN1786Asp & Yes \\
\hline 4 & 17 & Male & White British & 26.4 & c.10769delC & p.Thr3590LysfsX6 & $c .5356 A>G$ & p.Asn1786Asp & Yes \\
\hline 5 & 20 & Male & White British & 31.5 & $c 6526 C>T$ & p.Gln217X & $c 11101 C>T$ & pArg3701X & No \\
\hline 6 & 49 & Male & White British & 31.9 & c. $10483 C>T$ & p.Gln3495X & c.10775delC & P.Threo3592LysfsX6 & No \\
\hline 7 & 40 & Male & White British & 38.4 & c.1729delA & p.Arg577Glyfsx17 & c.10477C > T & p.Gln3493X & No \\
\hline 8 & 25 & Female & Mixed race & 30.0 & c.2218dupA & p.ThreoAsnfsX2 & c. $1069 C>T$ & p.Arg3657X & No \\
\hline 9 & 45 & Male & White British & 35.3 & c. $1874 A>G$ & p.His623Arg & & & No \\
\hline $10 \#$ & 44 & Male & White British & 28.2 & c.10769delC & p.Thr3590LysfsX6 & C. $11410 C>T$ & p.Arg38404X & Yes \\
\hline 11 & 21 & Female & White British & 26.4 & c6584delA & p.Lys2195SerfX10 & C1008_1009delT & pCys336fsX1 & Yes \\
\hline 12 & 22 & Female & White British & 27.6 & c. $6823 C>T$ & p.Arg2275X & $c .9535 C>T$ & P.Arg3179X & No \\
\hline 13 & 26 & Male & White British & 22.7 & c. $6823 C>T$ & p.Arg2275X & c. $9535 C>T$ & P.Arg3179X & No \\
\hline 14 & 29 & Male & White British & 31.6 & c.6895delG & p.val2299TrpsX43 & C.11443C > T & p.Gln3815X & No \\
\hline 15 & 43 & Male & White British & 35.2 & C. $8995 C>T$ & p.Gln3493X & $c>9001 C>T$ & Gln3001 & No \\
\hline 16 & 29 & Female & White British & 27.0 & $c .8002 C>T$ & p.arg2668X & c. $10879 C>T$ & pArg3627x & No \\
\hline 17 & 18 & Male & BritishPakistani & 27.8 & c.4937C > A & p.Ser1646X & & & No \\
\hline 18 & 20 & Male & BritishPakistani & 36.6 & c.7544-200_767+1110del & & & & No \\
\hline 19 & 19 & Male & BritishPakistani & 26.8 & c.7544-200_767+1110del & & & & Yes \\
\hline 20 & 21 & Female & BritishPakistani & 29.6 & c.5075delC & p.Pro1692LeufsX39 & c.5075delC & p.Pro1692LeufsX39 & Yes \\
\hline 21 & 24 & Female & White British & 26.5 & Result awaited* & - & - & - & No \\
\hline 22 & 20 & Male & BritishPakistani & 25.9 & c.4937C > A & p.Ser2646STOP & & & Yes \\
\hline 23 & 19 & Female & British Indian & 19.4 & $c .2041 C>T$ & p.Arg681X & $c .2041 C>T$ & p.Arg681X & Yes \\
\hline 24 & 23 & Male & British Indian & 28.9 & c. $2041 C>T$ & p.Arg681X & c. $2041 C>T$ & p.Arg681X & No \\
\hline 25 & 32 & Female & White British & 25.7 & $C 11101 C>T$ & p.R3701X & & & No \\
\hline 26 & 20 & Female & White Australian & 37.7 & c.7911dupC & p.Asn2638Glnfs24 & & & No \\
\hline
\end{tabular}

\#End-stage kidney disease on dialysis

*Genetic data pending at time of publication. Patient fulfills the clinical diagnostic criteria for ALMS on serial assessment

for matched normal controls in the absence of LGE (Table 3). There was no difference in mean precontrast myocardial T1 times between ALMS and controls (Table 3). Indeed, 11 ALMS patients (41\%) had T1 times within the range for matched controls (943 ms). Post contrast T1 times were reduced in ALMS (Table 3).

\section{Late gadolinium enhancement}

Late gadolinium enhancement in a diffuse, non-coronary artery pattern was present in 9 (35\%) patients with ALMS (two with a history of infantile cardiomyopathy). An example is shown in Fig. 2. Compared to ALMS subjects with no LGE but increased ECV, subjects with LGE did not have increased ECV $(0.34 \pm 0.05$ vs. $0.31 \pm 0.03, p=0.22$ ) and no differences in LV volumes, ejection fraction or deformation (Table 3 ).

\section{ECG}

All ALMS patients were in sinus rhythm with normal atrial activation (PR interval $143 \mathrm{~ms} \pm 16$ ), ventricular depolarisation (QRS duration $88 \mathrm{~ms} \pm 16$ ) and ventricular repolarisation (QTc $416 \mathrm{~ms} \pm 24$ and $426 \mathrm{~ms} \pm 24$ for males and females respectively). Patients with ECV above the upper limit of the range for matched controls had increased QTc $(430 \pm 21 \mathrm{~ms}$ vs. $412 \pm 18 \mathrm{~ms}, \mathrm{p}<0.05)$.

\section{Myocardial fibrosis}

ECV correlated with age $(\mathrm{r}=0.50, \mathrm{p}<0.05)$, global longitudinal peak systolic strain $(\mathrm{r}=-0.73, \mathrm{p}<0.01)$, peak systolic strain rate $(r=-0.57, p<0.01)$, MAPSE $(r=-0.39, p<0.05)$, but not ejection fraction, NT-BNP or LV mass/height ${ }^{2.7}$. Serum triglycerides $(\mathrm{r}=0.66, \mathrm{p}<0.01)$ and QTc $(\mathrm{r}=0.49$, $\mathrm{p}<0.05$ ) also correlated with ECV (Fig. 3). These factors were entered into a multivariable linear regression model 
Table 2 Demographic, haemodynamic and biochemical data

\begin{tabular}{|c|c|c|}
\hline & Controls & ALMS \\
\hline Number $n$ & 26 & 26 \\
\hline Age years & $27 \pm 6$ & $27 \pm 9$ \\
\hline Male $n(\%)$ & $17(65 \%)$ & $17(65 \%)$ \\
\hline Height (m) & $1.74 \pm 0.09$ & $1.61 \pm 0.09^{* *}$ \\
\hline Weight (Kg) & $76 \pm 16$ & $77 \pm 16$ \\
\hline Body Mass Index & $25 \pm 4$ & $30 \pm 5^{* *}$ \\
\hline Haemoglobin (g/dL) & $14.3 \pm 1.2$ & $14.0 \pm 2.2$ \\
\hline $\operatorname{eGFR}\left(\mathrm{ml} / \mathrm{min} / 1.73 \mathrm{~m}^{2}\right)$ & $87 \pm 8$ & $73 \pm 25^{*}$ \\
\hline $\operatorname{Renin}(\mathrm{pg} / \mathrm{ml})^{a}$ & - & $41(18-153)$ \\
\hline Aldosterone $(\mathrm{pg} / \mathrm{ml})^{a}$ & - & $97(67-220)$ \\
\hline NT-pro BNP (ng/L) a & $38(4-75)$ & $37(17-89)$ \\
\hline Cholesterol (mmol/L) & $4.6(0.8)$ & $5.1 \pm 1.3$ \\
\hline Triglycerides $(\mathrm{mmol} / \mathrm{L})^{a}$ & $1.0(0.6-1.4)$ & $2.5(1.5-4.1)^{* *}$ \\
\hline High Density Lipoprotein (mmol/L) & $1.4(0.3)$ & $0.9 \pm 0.3^{* *}$ \\
\hline Glucose $(\mathrm{mmol} / \mathrm{L})^{a}$ & $4.7(4.2-5.0)$ & $6.1(4.6-15.0)^{*}$ \\
\hline $\mathrm{HbA}_{1 \mathrm{c}}(\%)$ & - & $7.9 \pm 2.7$ \\
\hline Insulin (pmol/L) ${ }^{a}$ & - & $858(239-1760)$ \\
\hline C-peptide:Glucose ratio (ng/ml:mg/dl) a & - & $4.2(2.2-6.2)$ \\
\hline $\mathrm{ACR}(\mathrm{mg} / \mathrm{mmol})^{a}$ & $0.1(0.05-0.2)$ & $1.5(0.25-34)$ \\
\hline Urate $(\mathrm{mmol} / \mathrm{L})$ & $286 \pm 67$ & $409 \pm 111^{*}$ \\
\hline $24 \mathrm{~h}$ daytime SBP $(\mathrm{mmHg})$ & $119 \pm 9$ & $130 \pm 14^{*}$ \\
\hline $24 \mathrm{~h}$ daytime DBP $(\mathrm{mmHg})$ & $72 \pm 7$ & $77 \pm 9^{*}$ \\
\hline
\end{tabular}

$A C R$ albumin/creatinine ratio, $A L M S$ Alström Syndrome, DBP diastolic blood pressure, eGFR Estimated glomerular filtration rate, $H B A_{1 c}$ glycated haemoglobin, NT-pro BNP N-terminal pro B natriuretic peptide, SBP systolic blood pressure

Value \pm standard deviation, ${ }^{a}$ median (inter-quartile range), $p={ }^{*}<0.05$, $p=* *<0.01$

with ECV as the dependent variable; $\mathrm{r}^{2}=0.75, \mathrm{p}<0.01$ Global longitudinal strain remained an independent predictor of ECV (unstandardized $\beta$ coefficient $-0.01,95 \%$ CI -0.02-0.004, $\mathrm{p}<0.01)$.

\section{Patient events}

Two patients (Table 1: patient 7 aged 40 and patient 10 aged 44 years) died over the study period. Both deaths occurred unexpectedly following a short respiratory tract infection which led to cardiac decompensation and multi-organ failure in the intensive care setting. Neither patient had a history of infantile cardiomyopathy but both had a history of severe hypertriglyceridaemia in teenage years $(>50 \mathrm{mmol} / \mathrm{l})$ with pancreatitis and raised plasma renin (>3000 $\mathrm{ng} / \mathrm{L})$ when normotensive. Furthermore, both had extensive patchy LGE on CMR, elevated global ECV (0.33 and 0.41 respectively) and reduced myocardial deformation despite a normal LV ejection fraction. On autopsy of one patient, there was extensive fibrosis in a non-ischaemic pattern in the RV which corresponded with the extensive LGE on CMR (Fig. 4) and evidence of a chronically occluded right coronary artery with extensive collateralisation.

\section{Discussion}

Interstitial myocardial fibrosis assessed using CMR T1 mapping, was evident in over $50 \%$ of patients with ALMS. Coarse fibrosis within the LV myocardium was detected in keeping with previous reports but a further $20 \%$ of patients were shown to have interstitial fibrosis missed using conventional LGE alone. Expansion of the ECV was associated with impaired global LV systolic deformation, impaired LV diastolic function and increased QT interval.. Impairment of global longitudinal strain and strain rate preceded any change in ejection fraction and without abnormality of conventional markers of elevated LV end-diastolic pressure and atrial stretch, including both NT pro-BNP and left atrial volume. This finding raises the possibility that surveillance of ALMS for evidence of cardiomyopathy using NT pro-BNP may be inadequate. In contrast to previous echocardiographic studies [24] that have reported 'low-normal' LV ejection fraction, use of CMR in young adults with ALMS indicates that patients have equivalent conventional values of ventricular size and EF to healthy controls but reduced myocardial deformation and diastolic function. These data support the concept that expansion of the ECV and reduced myocardial deformation may be more powerful predictors of adverse cardiovascular outcome than ejection fraction [22].

Although ALMS is a rare autosomal recessive genetic disorder, there is much interest in understanding the disease as a monogenic model for end-organ fibrosis which has been proven to affect the liver and kidneys [4]. The increase in diffuse interstitial myocardial fibrosis (as measured by ECV) and prolongation of the QTc interval on 12 lead electrocardiograms in this study are consistent with the limited autopsy data available in 5 ALMS patients in whom moderate to severe interstitial myocardial fibrosis was found [9]. The data are also consistent with a smaller study of 8 patients, only 3 of whom had normal LVEF at the time of CMR, in which T1 scout sequences were used to identify lower pre-contrast $\mathrm{T} 1$ relaxation as a measure of diffuse fibrosis [10]. Further studies using T1-mapping have demonstrated that diffuse interstitial fibrosis is prominent in diabetic patients [25], correlates with insulin resistance [26] and is associated with adverse prognosis [27]. In keeping with these data, our ALMS cohort displayed shorter myocardial post contrast T1 times and reduced deformation which is not unexpected given the high prevalence of impaired glycaemia. Furthermore, the correlation between expansion of the ECV and elevated triglycerides in our study may be consistent with poor diabetic control and 
Table 3 Cardiac MRI Functional and Fibrosis data

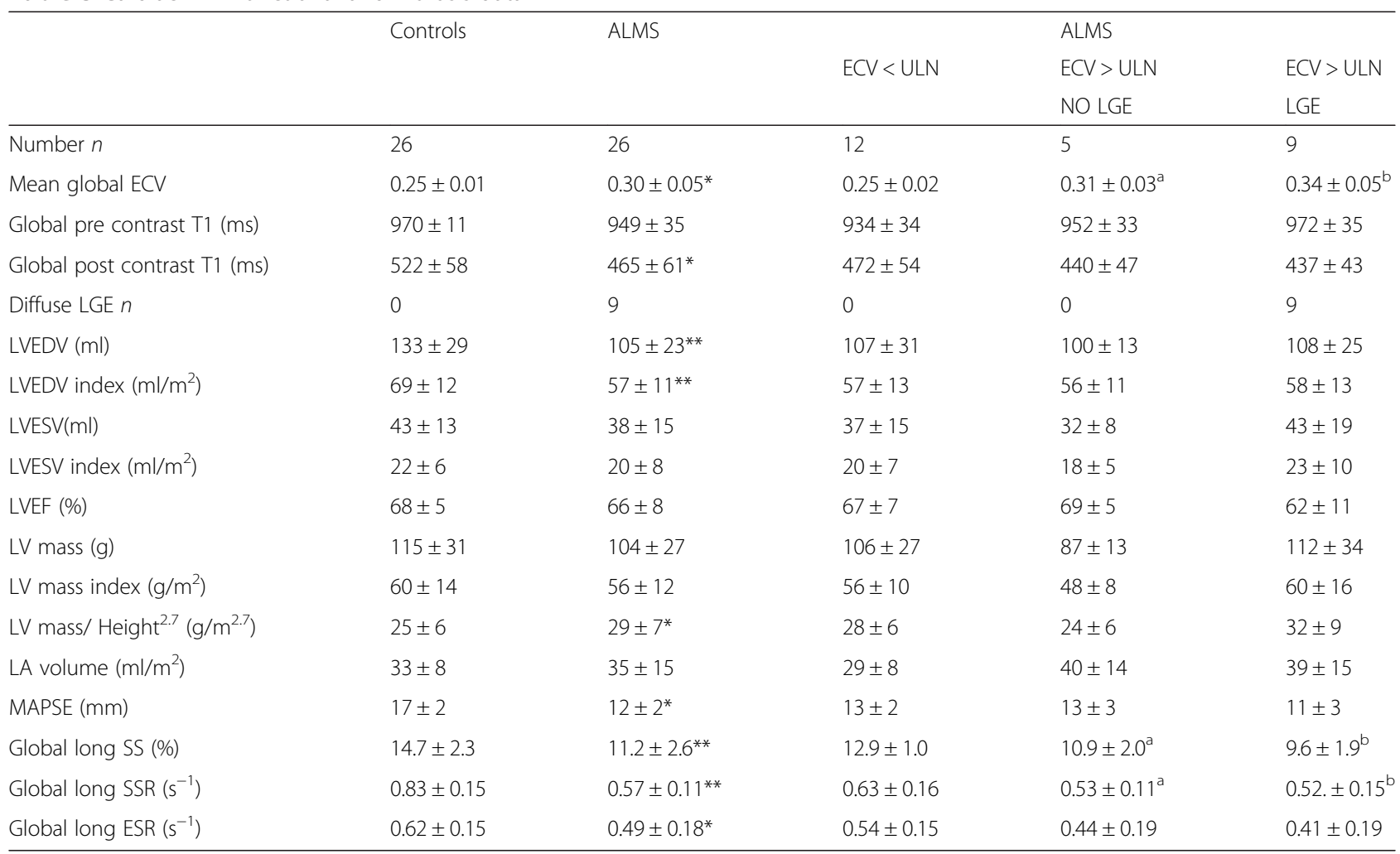

EDV end-diastolic volume, ESV end-systolic volume, EF ejection fraction, $L A$ left atrium, $L G E$ late gadolinium enhancement, long; longitudinal, $L V$ left ventricle, MAPSE mitral annular plane systolic excursion, $R V$ right ventricle, TAPSE tricuspid annular plane systolic excursion, SS systolic strain, SR systolic strain rate, ESR early diastolic strain rate, ULN ECV above the upper limit (0.279) observed in matched control

${ }^{a} p<0.05$, Bonferroni post-hoc tests for differences ECV above upper limit of normal controls vs. increased ECV no LGE

${ }^{\mathrm{b}} p<0.05$, Bonferroni post-hoc tests for differences ECV above upper limit of normal controls vs. increased ECV with LGE

ANOVA * $p<0.05, * *<0.01$, ALMS vs. controls

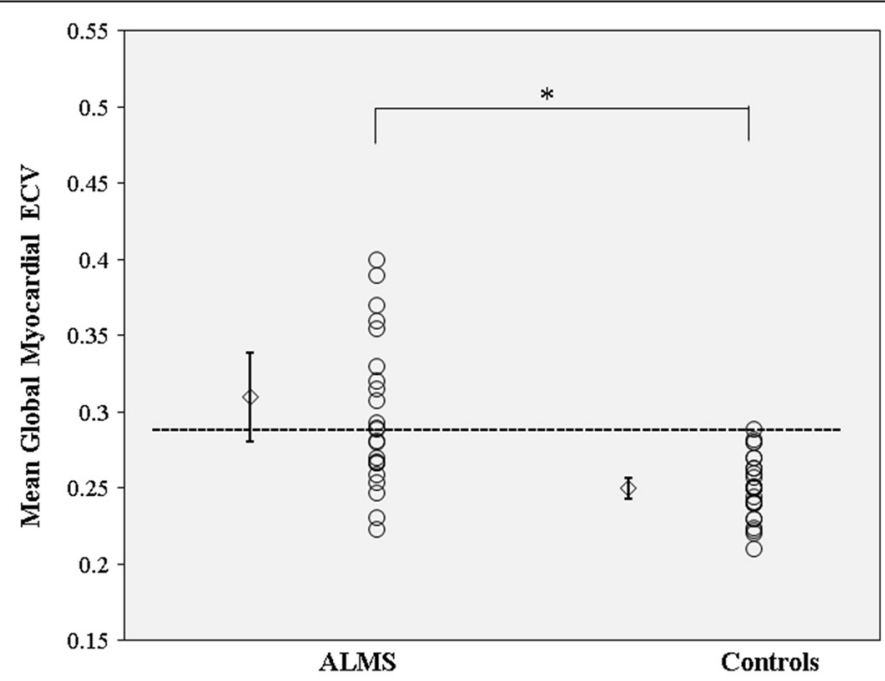

Error Bars \pm 2 SEM

Fig. 1 Box scatter plots of global extracellular volume (ECV) in patients with ALMS and controls. Error bars are standard error of the mean $\times 2$. Dashed horizontal line defines the upper value within the matched normal population $(0.279)$. ECV was assessed in the left ventricle from the basal and mid ventricular levels and averaged to yield a "global ECV" measurement. * $p<0.05$ 

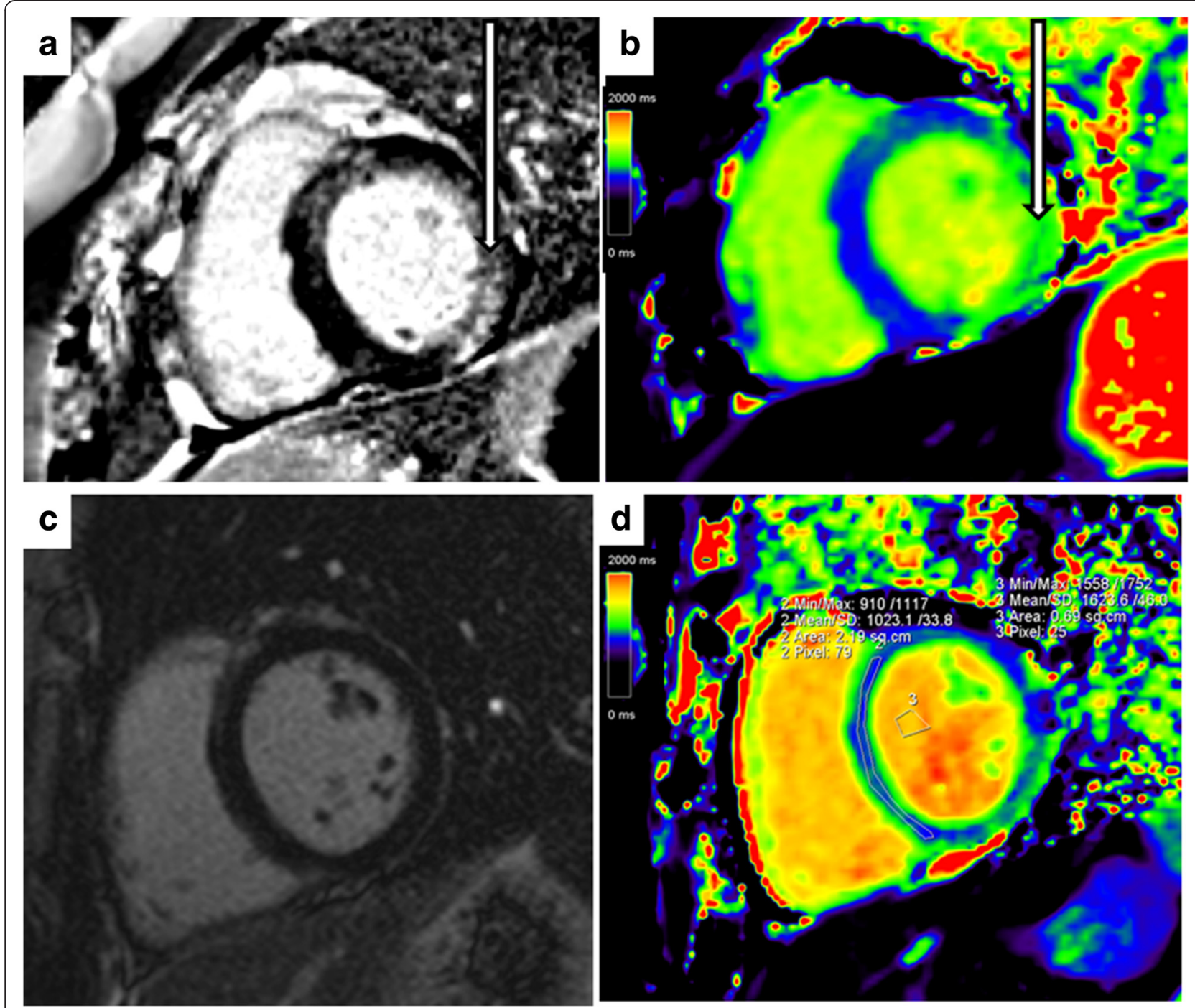

Fig. 2 Examples of late gadolinium enhancement and corresponding pre-contrast colour T1 maps in patients with ALMS. a Clear late enhancement (arrow) in the basal infero-lateral wall. b Corresponding high T1 (1057 ms) on the T1-map (arrow). c No late enhancement in the left ventricular myocardium. d Corresponding areas with high T1 (1043 ms) on the T1-map in the septum and infero-lateral wall (green)

supports the latter as a potential mediator. The mechanism behind the development of fibrosis within the myocardium is not known. It has recently been shown that ALMS1 is an important molecule for cell cycle regulation in perinatal cardiomyocytes and that deficiency of the protein may lead to a mitogenic cardiomyopathy [28]. This was supported by the histological diagnosis of mitogenic cardiomyopathy in two siblings from consanguineous parents of Turkish origin who died in infancy from dilated cardiomyopathy [29]. This may explain perinatal mortality but other mechanisms may be important in the development of adult cardiomyopathy, such as the onset of adiposopathy, wherein adiposite enlargement may be associated with inflammation and insulin resistance [30].
We acknowledge that while mean global ECV was higher than controls in this study, expansion of the ECV was not universal and there was considerable dispersion of ECV values within the range of normal healthy controls. Indeed, $40 \%$ of patients had a native precontrast myocardial $\mathrm{T} 1$ values below the cut off for healthy volunteers $(943 \mathrm{~ms})$ raising the possibility of myocardial fat deposition or pseudonormalization of $\mathrm{T} 1$ due to the combined presence of lipid and fibrosis within the LV. This significant inter-individual variation suggests that development of fibrosis is not an inevitable consequence of the genetic defect alone and that environmental factors may also play an important role. Further serial studies are warranted in ALMS to determine whether those with higher ECV are susceptible to adverse 

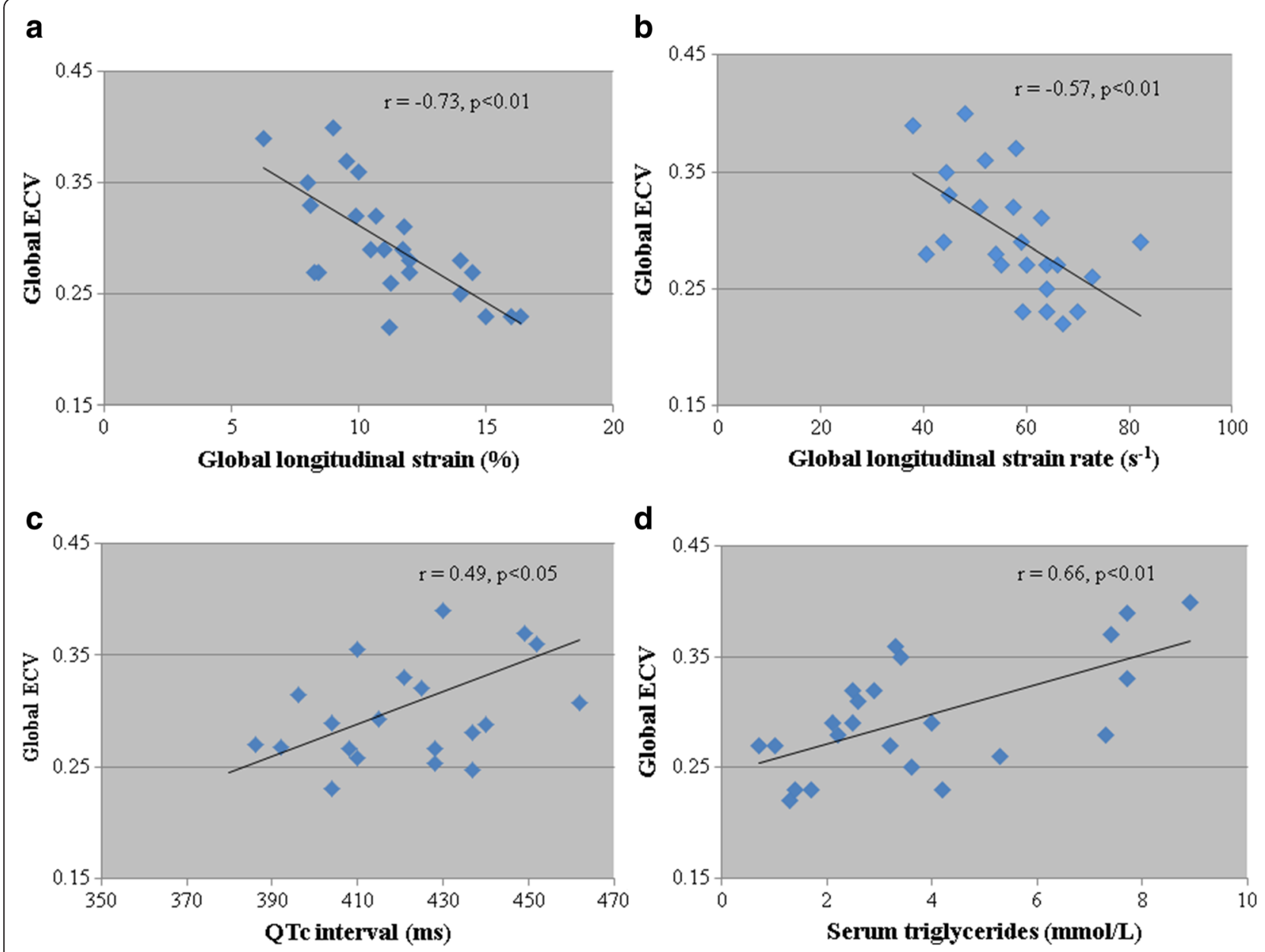

Fig. 3 Association of diffuse myocardial fibrosis with markers of lipid metabolism, systolic function and cardiac electrical activity. Scatter plots demonstrating the association between global extracellular volume and a) global longitudinal strain, b) global systolic strain rate, c) 12 lead ECG QTc interval and $\mathbf{d}$ ) serum triglycerides in patients with ALMS

cardiovascular outcomes, as has been shown to be the case in other populations [27].

Despite the fact that ALMS patients are characterised by obesity, severe insulin resistance and metabolic syndrome $[13,2]$, reduction in myocardial deformation was not due to myocardial infarction, since the distribution of LGE was neither subendocardial nor consistent with a coronary artery territory. It is possible that expansion of the ECV with increased diastolic stiffness in association with subclinical ventricular dysfunction [31] may have contributed to the failure of the two adult patients to survive intercurrent respiratory tract infections, as occurred in this series. It is also interesting to speculate whether expansion of the $\mathrm{ECV}$ is responsible for prolongation of the QTC and whether this predisposes individuals to lethal arrhythmia. It is noteworthy that despite extensive fibrosis in the two patients that died, both were asymptomatic before developing a chest infection.
An unusual feature of ALMS is the occurrence of infantile cardiomyopathy within the first few months of life which, if not fatal during the acute stage, may be followed by an apparent complete recovery [8]. Adults with ALMS have an increased risk of adverse cardiovascular outcomes but this risk does not appear to be heightened by a history of infantile cardiomyopathy. In our study, there was no relationship between exposure to infantile $\mathrm{CM}$ and the severity of diffuse interstitial fibrosis, presence of coarse scarring on LGE or reduction either in LVEF or in myocardial deformation. These data are consistent with the theory that the pathophysiology of early (infantile) and late cardiovascular morbidity and mortality is different. Coarse replacement fibrosis documented with LGE does not appear to be simply a consequence of scarring from severe cardiomyopathy in childhood, a concept supported by two previous smaller studies in ALMS [11, 14]. In addition, the recent work by Shenje et al. [28] and Louw et al. [29] have suggested a 


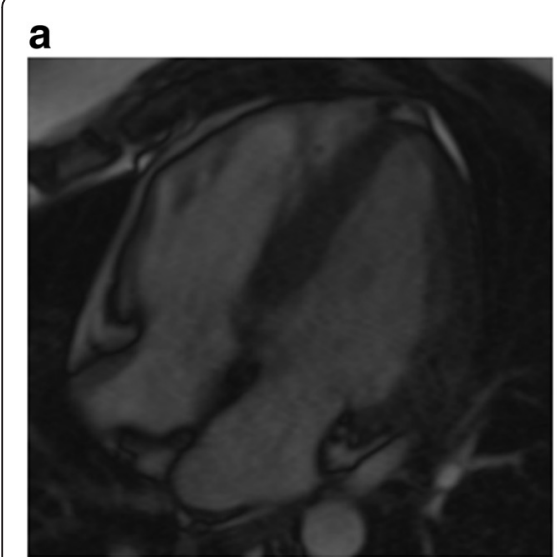

b

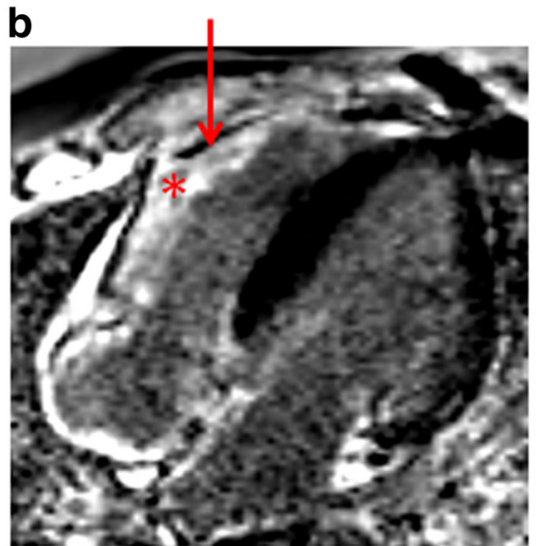

d

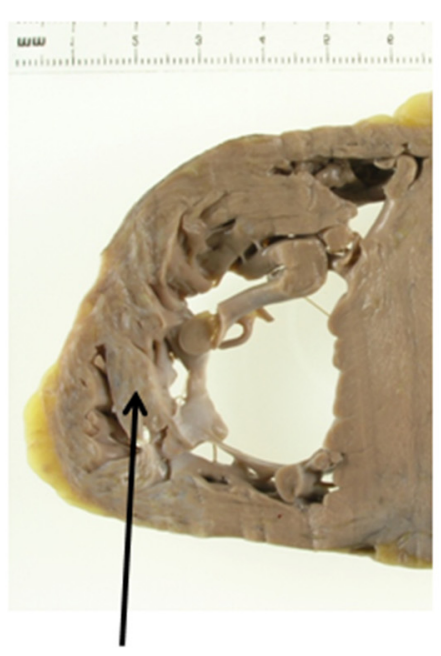

e

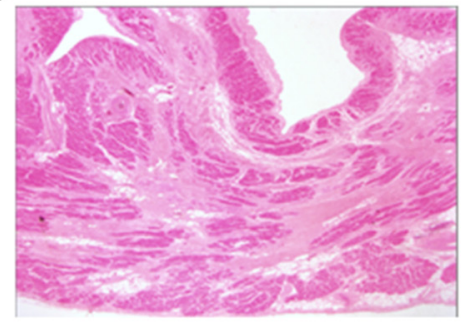

f

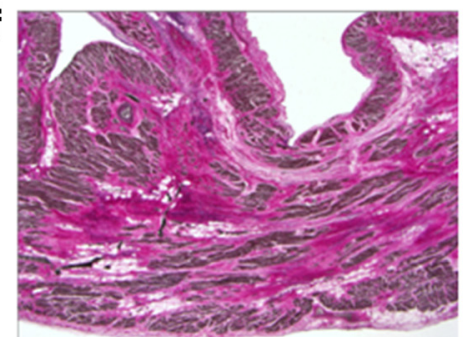

c

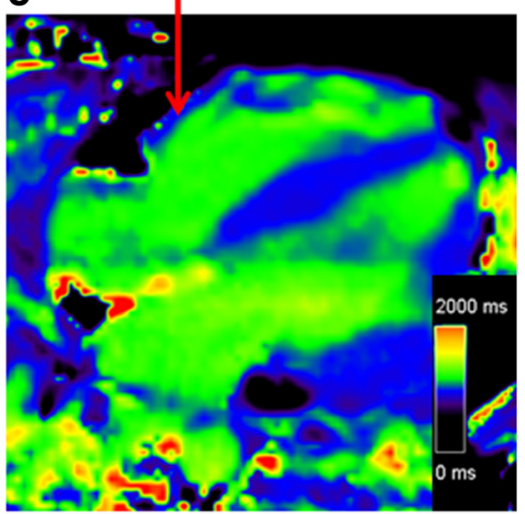

g

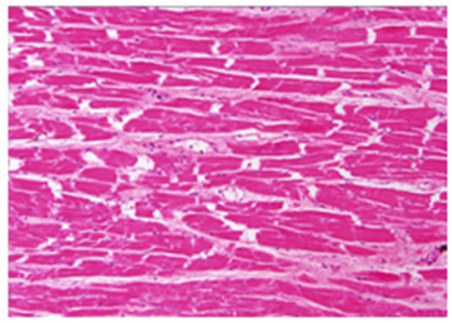

h

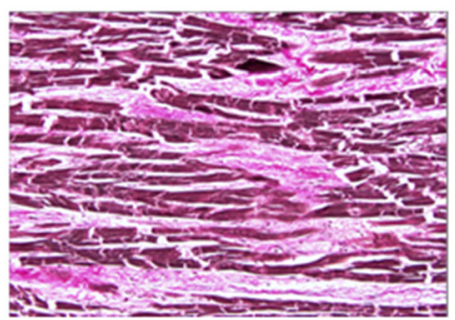

Fig. 4 Myocardial fibrosis on CMR and post-mortem specimens from a patient with ALMS. a-c) 4 chamber CMR imaging; a cine, b late gadolinium enhancement and c T1 mapping. Note the extensive LGE in the RV $\left(^{*}\right)$ with corresponding low T1 on the post-contrast MOLLI. d Corresponding macroscopic image from autopsy of the RV with strands of sub-endocardial fibrosis seen as pallor within the RV (black arrow). e-f Corresponding low power views of part of the free wall of the right ventricle demonstrating patchy swathes of interstitial fibrosis replacing myocardiocytes with more subtle pericellular fibrous expansion. The fibrous tissue stains pink with H\&E and red with EHVG (H\&E \& EHVG, original magnification X1.25). $\mathbf{g}$-h Comparable intermediate magnification photomicrographs of the free wall of the right ventricle depicting more dispersed interstitial fibrosis enclaving groups of myocytes and focally insinuating between individual muscle cells. The fibrous tissue stains pink with H\&E and red with EHVG (H\&E stain, original magnification X1.25)

mitogenic cardiomyopathy may underly infantile dilated cardiomyopathy, although these changes have not been documented in adult post-mortem hearts. Inferences relating to infantile cardiomyopathy from our data however are limited by the fact that our study only included adult survivors, since patients under the age of 16 years are excluded from referral to the UK National Centre at Queen Elizabeth Hospital.

\section{Limitations}

Given the small sample size and narrow age range of patients with this rare disease, we were underpowered to show some relationships reported previously in ECV
[32]. This is a cross-sectional, observational study, and hence it is only possible to speculate on the aetiology of diffuse fibrosis and whether it is represents an intermediate phenotype in ALMS between cardiovascular health, coarse myocardial fibrosis and ultimately LV failure. The extent to which diffuse interstitial fibrosis is related to the primary effect of the ALMS1 mutation on fibroblast expression or to the severe metabolic disturbance warrants further study [33, 34]. Due to the rarity of this condition, there are relatively few available patients to study and echocardiography is particularly limited by poor acoustic windows in ALMS patients. In our experience, CMR scanning takes longer and requires practice of breath 
holding techniques but is feasible in the great majority despite most adult ALMS patients having both severe hearing and visual impairment. Biomarkers would be of particular use in ALMS given the limitations of echocardiography and the limited availability of CMR but standard markers of cardiovascular stress, including NT pro-BNP were not useful. Future studies should consider the measurement of direct markers of collagen turnover and fibrosis, such as serum C-terminal propeptide of type I pro-collagen [35].

\section{Conclusion}

Diffuse interstitial fibrosis is common in asymptomatic patients with ALMS and is associated with abnormalities of LV systolic and diastolic function. The presence of cardiac fibrosis may be actively modulated by the severity of metabolic dysfunction, with elevated triglycerides a potential marker or intermediary. Expansion of the ECV is an imaging biomarker of cardiovascular vulnerability that demands longitudinal study in ALMS.

\section{Competing interests}

The authors declare that they have no competing interests.

\begin{abstract}
Authors' contribution
All authors have made substantial contributions to the conception, design, acquisition, analysis or interpretation of data. NE, RBP, RPS, and TG conceived this study. NE, WEM, and MY were responsible for the data acquisition and analysis. ATW was responsible to histopathological analysis and RC for supervising biochemical analysis. NE and RPS were responsible for the first version of the manuscript but all authors have contributed to subsequent versions and have approved the final version of the submitted manuscript.
\end{abstract}

\section{Acknowledgements}

Alström Syndrome UK, James Hodson for statistical support.

\section{Funding}

Participation of healthy control subjects was funded through an on-going British Heart Foundation research study (FS/11/17/28700).

\section{Data}

All data is will be made available in the open access The University of Birmingham Research Archive [UBIRA] repository.

\section{Author details}

${ }^{1}$ School of Clinical and Experimental Medicine, University of Birmingham, Birmingham, UK. ${ }^{2}$ Department of Cardiology, Queen Elizabeth Hospital, Birmingham, UK. ${ }^{3}$ Department of Pathology, Queen Elizabeth Hospital, Birmingham, England. ${ }^{4}$ Department of Biochemistry, Queen Elizabeth Hospital, Birmingham, UK. ${ }^{5}$ Department of Diabetes, Torbay Hospital, Torbay, UK. ${ }^{6}$ Department of Endocrinology, Queen Elizabeth Hospital, Birmingham, UK.
}

Received: 21 March 2015 Accepted: 4 June 2015 Published online: 24 June 2015

\section{References}

1. Alstrom $\mathrm{CH}$, Hallgren $\mathrm{B}$, Nilsson LB, Asander $\mathrm{H}$. Retinal degeneration combined with obesity, diabetes mellitus and neurogenous deafness: a specific syndrome (not hitherto described) distinct from the Laurence-Moon-Bardet-Biedl syndrome: a clinical, endocrinological and genetic examination based on a large pedigree. Acta Psychiatr Neurol Scand Suppl. 1959;129:1-35.

2. Bettini V, Maffei P, Pagano C, Romano S, Milan G, Favaretto F, et al. The progression from obesity to type 2 diabetes in Alstrom syndrome. Pediatr Diabetes. 2012;13:59-67.
3. Marshall JD, Muller J, Collin GB, Milan G, Kingsmore SF, Dinwiddie D et al. Alstrom Syndrome: Mutation Spectrum of ALMS1. Hum Mutat. 2015. In press.

4. Marshall JD, Maffei P, Collin GB, Naggert JK. Alstrom syndrome: genetics and clinical overview. Curr Genomics. 2011;12:225-35.

5. Minton JA, Owen KR, Ricketts CJ, Crabtree N, Shaikh G, Ehtisham S, et al. Syndromic obesity and diabetes: changes in body composition with age and mutation analysis of ALMS1 in 12 United Kingdom kindreds with Alstrom syndrome. J Clin Endocrinol Metab. 2006;91:3110-6.

6. Marshall JD, Paisey RB, Carey C, Macdermott S. EditorsIn: Pagon RA, Adam MP, Ardinger HH, Wallace SE, Amemiya A, Bean LJH, Bird TD, Dolan CR, Fong CT, Smith RJH, Stephens K, editors. Source GeneReviews ${ }^{\oplus}$ [Internet]. Seattle (WA): University of Washington, Seattle; 1993-2015. 2003 Feb 7 [updated 2012 May 31].

7. Marshall JD, Beck S, Maffei P, Naggert JK. Alstrom syndrome. Eur J Hum Genet. 2007;15:1193-202

8. Michaud JL, Heon E, Guilbert F, Weill J, Puech B, Benson L, et al. Natural history of Alstrom syndrome in early childhood: onset with dilated cardiomyopathy. J Pediatr. 1996;128:225-9.

9. Marshall JD, Bronson RT, Collin GB, Nordstrom AD, Maffei P, Paisey RB, et al. New Alstrom syndrome phenotypes based on the evaluation of 182 cases. Arch Intern Med. 2005;165:675-83.

10. Corbetti F, Razzolini R, Bettini V, Marshall JD, Naggert J, Tona F, et al. Alstrom syndrome: cardiac magnetic resonance findings. Int J Cardiol. 2013;167:1257-63.

11. Loudon MA, Bellenger NG, Carey CM, Paisey RB. Cardiac magnetic resonance imaging in Alstrom syndrome. Orphanet J Rare Dis. 2009;4:14.

12. Jellis C, Martin J, Narula J, Marwick TH. Assessment of nonischemic myocardial fibrosis. J Am Coll Cardiol. 2010;56:89-97.

13. Paisey RB. New insights and therapies for the metabolic consequences of Alstrom syndrome. Curr Opin Lipidol. 2009;20:315-20.

14. Paisey RB, Carey CM, Bower L, Marshall J, Taylor P, Maffei $P$, et al. Hypertriglyceridaemia in Alstrom's syndrome: causes and associations in 37 cases. Clin Endocrinol. 2004;60:228-31.

15. Paisey RB, Hodge D, Williams K. Body fat distribution, serum glucose, lipid and insulin response to meals in Alstrom syndrome. J Hum Nutr Diet. 2008;21:268-74.

16. Moody WE, Tomlinson LA, Ferro CJ, Steeds RP, Mark PB, Zehnder D, et al. Effect of A Reduction in glomerular filtration rate after NEphrectomy on arterial STiffness and central hemodynamics: rationale and design of the EARNEST study. Am Heart J. 2014;167:141-9.

17. Maceira AM, Prasad SK, Khan M, Pennell DJ. Normalized left ventricular systolic and diastolic function by steady state free precession cardiovascular magnetic resonance. J Cardiovasc Magn Reson. 2006;8:417-26.

18. Moody WE, Taylor RJ, Edwards NC, Chue CD, Umar F, Taylor TJ et al. Comparison of magnetic resonance feature tracking for systolic and diastolic strain and strain rate calculation with spatial modulation of magnetization imaging analysis. J Magn Reson Imaging. 2014 [Epub]

19. Schelbert EB, Testa SM, Meier CG, Ceyrolles WJ, Levenson JE, Blair AJ, et al. Myocardial extravascular extracellular volume fraction measurement by gadolinium cardiovascular magnetic resonance in humans: slow infusion versus bolus. J Cardiovasc Magn Reson. 2011;13:16.

20. de Simone G, Daniels SR, Devereux RB, Meyer RA, Roman MJ, de Divitiis O, et al. Left ventricular mass and body size in normotensive children and adults: assessment of allometric relations and impact of overweight. J Am Coll Cardiol. 1992;20:1251-60.

21. Ugander M, Oki AJ, Hsu LY, Kellman P, Greiser A, Aletras AH, et al. Extracellular volume imaging by magnetic resonance imaging provides insights into overt and sub-clinical myocardial pathology. Eur Heart J. 2012;33:1268-78.

22. Wong TC, Piehler K, Meier CG, Testa SM, Klock AM, Aneizi AA, et al. Association between extracellular matrix expansion quantified by cardiovascular magnetic resonance and short-term mortality. Circulation. 2012;126:1206-16.

23. Barna I, Keszei A, Dunai A. Evaluation of Meditech ABPM-04 ambulatory blood pressure measuring device according to the British Hypertension Society protocol. Blood Press Monit. 1998;3:363-8.

24. Makaryus AN, Zubrow ME, Marshall JD, Gillam LD, Mangion JR. Cardiac manifestations of Alstrom syndrome: echocardiographic findings. J Am Soc Echocardiogr. 2007;20:1359-63.

25. Ng AC, Auger D, Delgado V, van Elderen SG, Bertini M, Siebelink HM, et al. Association between diffuse myocardial fibrosis by cardiac magnetic resonance contrast-enhanced T(1) mapping and subclinical myocardial dysfunction in diabetic patients: a pilot study. Circ Cardiovasc Imaging. 2012;5:51-9. 
26. Jellis C, Wright J, Kennedy D, Sacre J, Jenkins C, Haluska B, et al. Association of imaging markers of myocardial fibrosis with metabolic and functional disturbances in early diabetic cardiomyopathy. Circ Cardiovasc Imaging. 2011;4:693-702.

27. Wong TC, Piehler KM, Kang IA, Kadakkal A, Kellman P, Schwartzman DS, et al. Myocardial extracellular volume fraction quantified by cardiovascular magnetic resonance is increased in diabetes and associated with mortality and incident heart failure admission. Eur Heart J. 2014;35:657-64.

28. Shenje LT, Andersen P, Halushka MK, Lui C, Fernandez L, Collin GB, et al. Mutations in Alstrom protein impair terminal differentiation of cardiomyocytes. Nat Commun. 2014;5:3416.

29. Louw JJ, Corveleyn A, Jia Y, lqbal S, Boshoff D, Gewillig M, et al. Homozygous loss-of-function mutation in ALMS1 causes the lethal disorder mitogenic cardiomyopathy in two siblings. Eur J Med Genet. 2014;57(9):532-5.

30. Favaretto F, Milan G, Collin GB, Marshall JD, Stasi F, Maffei P, et al. GLUT4 defects in adipose tissue are early signs of metabolic alterations in Alms1GT/GT, a mouse model for obesity and insulin resistance. PLoS One. 2014;9(10):e109540

31. Toulany A, Shea S, Warren AE. Doppler tissue, strain, and strain rate imaging in pediatric patients with Alstrom syndrome: are there regional functional abnormalities? J Am Soc Echocardiogr. 2006;19:14-20.

32. Liu CY, Liu YC, Wu C, Armstrong A, Volpe GJ, van der Geest RJ, et al. Evaluation of age-related interstitial myocardial fibrosis with cardiac magnetic resonance contrast-enhanced T1 mapping: MESA (Multi-Ethnic Study of Atherosclerosis). J Am Coll Cardiol. 2013;62:1280-7.

33. Romano S, Milan G, Veronese C, Collin GB, Marshall JD, Centobene C, et al. Regulation of Alstrom syndrome gene expression during adipogenesis and its relationship with fat cell insulin sensitivity. Int J Mol Med. 2008;21:731-6.

34. Zulato E, Favaretto F, Veronese C, Campanaro S, Marshall JD, Romano S, et al. ALMS1-deficient fibroblasts over-express extra-cellular matrix components, display cell cycle delay and are resistant to apoptosis. PLoS One. 2011;6:e19081.

35. Ho CY, Lopez B, Coelho-Filho OR, Lakdawala NK, Cirino AL, Jarolim P, et al. Myocardial fibrosis as an early manifestation of hypertrophic cardiomyopathy. N Engl J Med. 2010;363(6):552-63.

\section{Submit your next manuscript to BioMed Central and take full advantage of:}

- Convenient online submission

- Thorough peer review

- No space constraints or color figure charges

- Immediate publication on acceptance

- Inclusion in PubMed, CAS, Scopus and Google Scholar

- Research which is freely available for redistribution 